\title{
Good papers, bad papers
}

\author{
Yasutomo Fujii
}

(C) The Japan Society of Ultrasonics in Medicine 2012

I have been a member of the editorial board for 4 years now. It is my view that the role of board members is to serve as gatekeepers to maintain the quality of JSUM and to support budding researchers. Whatever they do, I do not think board members should ever call attention to themselves while needlessly misleading researchers with confusing behavior.

When I first accepted this position, I thought it would be a bit tedious, but being a board member has turned out to be quite enjoyable, with many things to absorb and the chance to learn things from both good papers and bad papers. Good papers are enjoyable to read, and I end up forgetting my role as I lose myself in the paper. Then there are the bad papers, the ones that are poor from start to finish, that irritate me and make my blood pressure rise. A bad paper does not necessarily mean that the author is a poor researcher. I look at it in a favorable light, assuming that there must be some underlying circumstance surrounding a single poor showing. Just what is a "bad paper"? I will describe below the most noteworthy problems I have observed during my 4 years as a board member.

\section{What is originality?}

Case reports consisting of only clinicopathologically rare cases (e.g., a histopathologically rare disease or a disease not common in children) may fall under this category. Such papers may be highly original, but they are probably not

\section{Y. Fujii ( $\square)$}

Department of Clinical Laboratory Medicine,

Jichi Medical University, 3311-1 Yakushiji,

Shimotsuke, Tochigi 329-0498, Japan

e-mail: yfujii@jichi.ac.jp suitable for a journal specializing in medical ultrasonics if a detailed comparison of clinicopathological findings and ultrasound images is absent. What role did ultrasound play in this case? Is the role it played really worthy of evaluation and reporting? I would like authors to think carefully about the answers to these questions before writing a paper. Papers written with the attitude that "anything is fine as long as ultrasound imaging is involved" are not worthy of being published.

\section{"So what?"}

I sometimes come across papers where I wonder what the author wants to say, like papers filled with a bunch of long citations from the literature and lacking any kind of conclusion. Are the authors trying to pad the paper to make it longer or wanting to brag about how much research they did? I do not know what their intention is, but either way they only succeed in confusing the reader. The abstract should be rational, simple, and straightforward.

\section{Ultrasound images that can hardly be called beautiful}

Publishing images that can hardly be called beautiful is a fatal error for a journal about diagnostic imaging. You should habitually pay attention to the frequency, focusing, and magnification when obtaining images.

\section{Unwieldy English}

Unlike papers written in Japanese, English manuscripts will most likely only be criticized when the English is 
really awful since the majority of reviewers at this journal are not native English speakers. However, there are reviewers out there who will take advantage of their anonymity to write a bunch of scathing negative criticisms, although surprisingly they sometimes write comments that miss the mark (for instance, there was a reviewer who insisted that the word "believe" should not be used in scientific papers). Therefore, it may be best to have your paper checked by a native English speaker with a scientific background before submitting it.

A major goal of the editorial board is to get our Englishlanguage Journal of Medical Ultrasonics listed on MEDLINE, and it is currently engaged in a variety of activities to achieve this goal. The most important (straightforward and also the most difficult) of these activities is to publish many "good papers." We earnestly await submissions of many good papers. 